\title{
Efeitos da Taxa de Ganho de Peso Pré-Desmama de Bezerras de Corte e do Nível Nutricional Pós-Parto, Quando Vacas, sobre a Produção e Composição do Leite e o Desempenho de Bezerros
}

\author{
João Restle1, Paulo Santana Pacheco², João Teodoro Padua ${ }^{3}$, José Luiz Moletta ${ }^{4}$, \\ Marta Gomes da Rocha ${ }^{5}$, José Henrique Souza da Silva ${ }^{6}$, Aline Kellermann de Freitas ${ }^{7}$
}

RESUMO - Foi estudada a taxa de ganho de peso de bezerras de corte na fase de aleitamento, do nascimento aos sete meses, e seu reflexo sobre o desempenho posterior, enquanto vacas. As taxas de ganho de peso avaliadas quando bezerras, durante o período prédesmame, foram: baixa (menor que $350 \mathrm{~g} / \mathrm{dia}$ ) e moderada (maior que $350 \mathrm{~g} / \mathrm{dia}$ ). Quando vacas e após o parto, foram mantidas com seus bezerros até o desmame, em pastagem nativa (PN) ou pastagem cultivada (PC). As vacas, quando bezerras, foram pesadas ao nascer e aos sete meses de idade (desmame) e, quando vacas, foram pesadas, com seus bezerros nas primeiras 24 horas pós-parto; aos 14, 42 , 70, 98, 126, 154, 182 dias; e ao desmame, que ocorreu com idade média dos bezerros de 228 dias. As avaliações da produção de leite e a coleta de amostras para avaliação da composição do leite foram realizadas nas mesmas datas das pesagens, com exceção do nascimento. Taxas de ganho de peso inferiores a $350 \mathrm{~g} /$ dia até os sete meses de idade não foram prejudiciais ao futuro peso das bezerras, em razão da compensação no peso que apresentaram na fase pós-desmama. Bezerras com taxas de ganho de peso menores que 350 g/ dia até os sete meses de idade, enquanto vacas produziram maiores quantidades de leite $(4,83 \mathrm{vs} 3,71 \mathrm{~L} / \mathrm{dia})$, total de gordura (47,4 vs $35,9 \mathrm{~kg}$ ), lactose (52,6 vs 39,7 kg), extrato seco total (140,4 vs 107,2 kg) e extrato seco desengordurado (94,8 vs 71,2 kg), e bezerros com maiores ganhos de peso ( $717 \mathrm{vs} 617 \mathrm{~g} / \mathrm{dia})$ e mais pesados ao desmame (189,0 vs 166,1 kg) que as vacas que, quando bezerras, apresentaram taxas de ganho de peso acima de $350 \mathrm{~g} / \mathrm{dia}$.

Palavras-chave: extrato seco total, ganho de peso, gordura, lactose, pastagem cultivada, pastagem nativa

\section{Preweaning Gain Rate of Beef Heifers and Postpartum Nutritional Level, as Cows, on Milk Production and Composition and Performance of their Calves}

\begin{abstract}
The preweaning weight gain rate of beef heifers and its effects on their subsequent performance as cows, was studied. The weight gain rates evaluated as heifer calves before weaning were low (below $350 \mathrm{~g} / \mathrm{day}$ ) and moderate (above $350 \mathrm{~g} / \mathrm{day}$ ). As cows and after calving, they were kept together with their calves until weaning on native (NP) or cultivated pasture (CP). The cows while heifers were weighted at birth and at weaning with seven months. As cows they were weighted with their calves $24 \mathrm{~h}$ after calving, at $14,42,70$, $98,126,182$ days postpartum, and at weaning when the calves reached average age of 228 days. The milk yield and the sampling for the evaluation of milk composition were taken at the same day of the weighing of the cows, with exception at birth. Weight rates below 350 $\mathrm{g} /$ day until seven months, did not affect the future weight of the heifers, due to compensatory gain after weaning. Heifers with weight gain rates below $350 \mathrm{~g} /$ day until seven month, as cows produced more milk (4.83 vs $3.71 \mathrm{~L} /$ day), total fat (47.4 vs $35.9 \mathrm{~kg}$ ), lactose (52.6 vs $39.7 \mathrm{~kg}$ ), total dry extract (140.4 vs $107.2 \mathrm{~kg}$ ) and nonfat dry extract (94.8 vs $71.2 \mathrm{~kg}$ ), calves with higher weight gain (717 vs 617 $\mathrm{g} /$ day) and heavier at weaning (189 vs $166.1 \mathrm{~kg}$ ), than cows that showed as heifer-calves weight gain rates above $350 \mathrm{~g} / \mathrm{day}$.
\end{abstract}

Key Words: total dry extract, weight gain, fat, lactose, cultivated pasture, native pasture

\section{Introdução}

Na produção de bovinos de corte, a produção de leite tem importância diferente da verificada em bovinos leiteiros, pois é destinada unicamente ao desenvolvimento do bezerro.
Diversos estudos demonstraram relação positiva entre produção de leite das vacas e desempenho dos bezerros, com coeficientes de correlação oscilando de 0,20 a 0,71 (Neville Jr., 1962; Melton et al., 1967; Rutledge et al., 1971; Chenette \& Frahm, 1981; Albuquerque et al., 1993). No estudo de Ribeiro \&

\footnotetext{
1 Engenheiro-Agrônomo, PhD, Pesquisador Visitante/CNPq - Departamento de Produção Animal - UFG. E.mail: jorestle@terra.com.br

2 Zootecnista, M.Sc., Aluno de Doutorado do Programa de Pós-Graduação em Ciência Animal da UFG - Goiânia, GO.

3 Zootecnista, Dr., Professor Adjunto do Departamento de Produção Animal da UFG.

4 Engenheiro-Agrônomo, M.Sc. Pesquisador IAPAR - PR.

5 Zootecnista, D.Sc., Professora Adjunto do Departamento de Zootecnia da UFSM.

6 Engenheiro-Agrônomo, PhD, Professor Titular do Departamento de Zootecnia UFSM.

7 Aluna de Mestrado do Programa de Pós-Graduação em Ciência Animal da UFG - Goiânia, GO. Bolsista do CNPq.
} 
Restle (1991), a produção de leite foi responsável por $56 \%$ da variação do peso do bezerro ao desmame. Bezerros mais pesados ao desmame são desejados em sistemas intensivos de produção, pois representam redução na idade de abate dos machos (Restle et al., 1999b) e redução na idade à puberdade das fêmeas (Restle et al., 1999a).

Além da quantidade de leite, a produção total dos componentes do leite também representa importância considerável, uma vez que são as principais fontes de nutrientes durante os primeiros meses de vida dos bezerros. Melton et al. (1967), Rutledge et al. (1971) e Totusek et al. (1973) verificaram que as produções dos componentes do leite apresentaram correlações altas e significativas com o desempenho dos bezerros até o desmame.

Portanto, compreender os fatores que interferem na produção e composição do leite e seus reflexos no desempenho dos bezerros são fundamentais para a pecuária bovina de corte. Segundo Ribeiro et al. (1991) e Quadros \& Lobato (1997), incrementar o nível nutricional é uma prática que pode ser empregada visando o aumento na produção de leite das vacas e o ganho de peso dos bezerros.

No entanto, estudos apontam para as consequiências que a velocidade de crescimento das bezerras pode causar em sua futura produção de leite, quando adultas. Elevadas taxas de ganhos de peso no período que antecede a puberdade podem resultar em menores produções de leite (Gardner et al., 1977; Ferrell, 1982; Johnsson \& Obst, 1984; Buskirk et al., 1996; Van Amburgh et al., 1998), causadas pelo acúmulo de tecido adiposo no úbere (Little \& Kay, 1979; Serjsen et al., 1982) e pela diminuição na quantidade de células mamárias (Hackett \& Tucker, 1968; Buskirk et al., 1996).

Foram avaliados, neste estudo, a influência de diferentes taxas de ganho de peso do nascimento até os sete meses de idade de bezerras e seu reflexo no desempenho subseqüente, quando vacas, na produção e composição de leite e no desempenho dos seus bezerros, em condições de pastagem cultivada ou pastagem nativa.

\section{Material e Métodos}

O experimento foi conduzido no Setor de Bovinocultura de Corte do Departamento de Zootecnia da Universidade Federal de Santa Maria, em Santa Maria - RS, localizado na região fisiográfica Depressão Central, a 153m de altitude, que, segundo classificação de Köppen, apresenta clima subtropical úmido (cfa) (Moreno, 1961). A temperatura média anual varia de
14,3 a $25,2^{\circ} \mathrm{C}$, com média das mínimas de $9,7^{\circ} \mathrm{C}$ no mês de agosto e média das máximas de $29,9^{\circ} \mathrm{C}$ no mês de janeiro, com possíveis geadas de abril a outubro. A média anual da umidade relativa do ar é de $73 \%$ e a precipitação é de 1650,9 mm (Rio Grande do Sul, 1979).

Foi estudada a influência da taxa de ganho de peso de bezerras de corte, na fase de aleitamento, do nascimento aos sete meses, e seu reflexo sobre o desempenho posterior, quando vacas. As taxas de ganho de peso avaliadas nos animais enquanto bezerras, durante o período pré-desmame foram: baixa (menor que $350 \mathrm{~g} /$ dia) e moderada (maior que $350 \mathrm{~g} /$ dia). Quando vacas e após o parto, foram mantidas com seus bezerros, até o desmame, em pastagem nativa (PN) ou pastagem cultivada (PC) de inverno (aveia + azevém) e de verão (capim-papuã). As taxas médias de ganho de peso das bezerras foram de $340 \pm 32$ e $490 \pm 29 \mathrm{~g}$, respectivamente, para os grupos baixa e moderada.

Foram utilizadas 28 vacas dos grupos genéticos Charolês $(\mathrm{CH})$ e Nelore (NE), que fizeram parte das 60 vacas estudadas por Restle et al. (2003), e das quais se tinham os dados referentes ao seu desenvolvimento na fase de aleitamento. Quando bezerras, foram pesadas ao nascer e aos sete meses de idade (desmame) e manejadas em pastagem nativa, juntamente com suas mães, até o desmame. No primeiro e segundo inverno, as bezerras tiveram acesso à pastagem cultivada (aveia + azevém), retornando, em seguida, para pastagem nativa até o momento da parição.

O período de parição, quando vacas, foi de setembro a dezembro, sendo os bezerros obtidos de inseminação artificial (período de 45 dias) e de monta natural (período de 45 dias).Durante o período de inseminação, foi utilizado o sêmen de oito touros $\mathrm{CH}$ e oito $\mathrm{NE}$ e, para o repasse, quatro touros $\mathrm{CH}$ e quatro touros $\mathrm{NE}$.

Para a pastagem cultivada, foi utilizada lotação/ha de 1,76 vacas com bezerro ao pé, tanto na PC de inverno como na de verão. Para os animais mantidos em PN, a lotação/ha foi de 1,0 vaca com bezerro ao pé.

Avaliou-se a massa de forragem disponível pela técnica da dupla amostragem (Wilm et al., 1944). Foram coletadas amostras da forragem a cada quatro semanas aproximadamente, cortadas rente ao solo, em uma área de $0,25 \mathrm{~m}^{2}$, para posterior determinação da matéria seca (MS) em estufa de ar forçado a $55^{\circ} \mathrm{C}$ por 72 horas, digestibilidade in vitro da matéria orgânica (DIVMO), conforme Tilley \& Terry (1963), e da proteína bruta (PB), pelo método micro kjeldahl, segundo AOAC (1984). 
No período de lactação, vacas e bezerros foram pesados nas primeiras 24 horas pós-parto, aos 14, 42, $70,98,126,154$ e 182 dias e ao desmame, que ocorreu com idade média dos bezerros de 228 dias.

As avaliações da produção de leite foram realizadas nas mesmas datas das pesagens, com exceção do nascimento. $\mathrm{O}$ método empregado para determinação da produção de leite foi o direto, com ordenha manual, segundo metodologia descrita por Ribeiro et al. (1991) e Restle et al. (2003).

Para avaliação da composição do leite, foram coletadas amostras de $300 \mathrm{~mL}$, após a ordenha, que foram enviadas imediatamente ao laboratório para determinação dos percentuais de gordura, lactose, extrato seco total e extrato seco desengordurado. A produção de leite foi corrigida para $4 \%$ de gordura, utilizando-se a seguinte equação: $\mathrm{L} 4 \%=0,4 \mathrm{P}+0,15 \mathrm{P} * \% \mathrm{G}$, em que: $\mathrm{L} 4 \%=$ produção de leite corrigida; $\mathrm{P}=$ produção de leite (L/dia) e $\% \mathrm{G}=$ porcentagem de gordura do leite.

$\mathrm{O}$ delineamento experimental adotado foi o inteiramente casualizado e os dados foram submetidos à análise de variância e aos testes $\mathrm{F}$ e Tukey, quando o F foi significativo a 5\%, por intermédio do programa estatístico SAS (1997). As variáveis também foram submetidas à análise de correlação de Pearson, empregando-se o seguinte modelo matemático:

$$
\begin{aligned}
\Upsilon_{i j k l m}= & \mu+\mathrm{NT}_{i}+\mathrm{IV}_{j}+\mathrm{PAST}_{k}+\mathrm{TXGANHO}_{l}+ \\
& \left(\mathrm{PAST}^{*} \mathrm{TXGANHO}_{k l}+\mathrm{E}_{i j k l m},\right.
\end{aligned}
$$

em que $\Upsilon_{i j k l m}=$ variáveis dependentes; $\mu=$ média geral de todas as observações; $\mathrm{NT}_{i}=$ efeito da covariável "ordem de nascimento do bezerro" de ordem "i”"; $\mathrm{IV}_{j}=$ efeito da covariável idade da vaca de ordem " j"; $\mathrm{PAST}_{k}=$ efeito do tipo de pastagem de ordem "k", sendo 1 =pastagem nativa; $2=$ pastagem cultivada; $\mathrm{TXGANHO}_{l}=$ efeito da taxa de ganho do nascimento aos sete meses de idade da vaca de ordem "l", sendo $1=$ baixa (menor que $0,350 \mathrm{~kg} /$ dia) e $2=$ moderada (maior ou igual a $0,350 \mathrm{~kg} / \mathrm{dia}$ ); $\left(\mathrm{PAST}^{*} \mathrm{TXGANHO}\right)_{k l}=$ interação entre k-ésimo sistema de alimentação e l-ésima taxa de ganho de peso da vaca; $\mathrm{E}_{i j k l m}=$ erro aleatório residual, $\operatorname{NID}\left(0, \sigma^{2}\right)$.

Análises preliminares comprovaram não haver diferença entre os grupos genéticos das vacas e, portanto, o grupo genético da vaca não foi incluído no modelo final.

O efeito do tipo de pastagem foi removido do modelo para as variáveis peso da vaca aos sete meses e ao parto e peso do bezerro ao nascer.

\section{Resultados e Discussão}

Durante todo o período experimental, os animais manejados em pastagem cultivada tiveram à disposi-

Tabela 1 - Médias para produção de leite (litros) de acordo com a taxa de ganho de peso da vaca em seu período pré-desmame e com o tipo de pastagem durante a lactação

Table 1 - Means for milk yield (litres) according to cow rate of weight gain in their preweaning period and with pasture during their lactation

\begin{tabular}{lcc}
\hline Pastagem & \multicolumn{2}{c}{ Taxa de ganho de peso quando bezerra $(\mathrm{kg})$} \\
Pasture & Rate of weight gain as heifer calf $(\mathrm{kg})$ \\
\cline { 2 - 3 } & Moderada & Baixa \\
& Moderate & Low \\
\hline
\end{tabular}

Produção diária de leite quando vaca (L)

Nativa (Native)

Cultivada (Cultivated)

Média (Mean)

\begin{tabular}{lr}
\multicolumn{2}{c}{ Daily milk yield as cow $(L)$} \\
3,46 & 4,60 \\
3,96 & 5,06 \\
$3,71 \mathrm{~B}$ & $4,83 \mathrm{~A}$
\end{tabular}

Produção total de leite (L) Total milk yield $(L)$

\begin{tabular}{lcc} 
Nativa (Native) & 750,2 & 998,9 \\
Cultivada (Cultivated) & 860,4 & 1097,4 \\
Média (Mean) & $805,3 \mathrm{~B}$ & $1048,2 \mathrm{~A}$ \\
\hline A, B Médias seguidas por letras maiúsculas diferentes na linha, para a mesma característica, diferem \\
\multicolumn{2}{c}{ ( $\mathrm{P}<0,05)$ pelo teste $\mathrm{F}}$. & \\
A, B Means followed by different capital letters in the line, for the same characteristic, differ $(P<.05)$ by $F$ test.
\end{tabular}


ção forragem de melhor qualidade, com superiores percentuais de proteína bruta $(7,9 \%)$ e digestibilidade in vitro da matéria orgânica $(59,2 \%)$ em relação à pastagem nativa (5,6 e 36,8\%, respectivamente).

$\mathrm{Na}$ Tabela 1, encontram-se os valores médios referentes às produções diária e total de leite, de acordo com a taxa de ganho de peso da vaca no seu período pré-desmame e com o tipo de pastagem durante sua lactação.

Bezerras com menor taxa de ganho de peso até os sete meses de idade, quando vacas apresentaram maiores produções diária (4,83 vs 3,71 L/dia) e total (1048,2 vs 805,3 L) de leite, que aquelas com maior taxa de ganho de peso. O ganho de peso médio diário nas bezerras com taxa ganho de peso considerada baixa, foi $31 \%$ inferior ao das bezerras com taxa de ganho de peso considerada moderada (340 vs $490 \mathrm{~g} /$ dia), resultando em $30 \%$ a mais de leite produzido quando vacas. No presente estudo, a correlação entre o ganho de peso até os sete meses de idade da vaca e produção de leite subseqüente foi de -0,19. Buskirk et al. (1996) relataram correlação de -0,28. De acordo com estes autores e com Hackett \& Tucker (1968), a produção de leite é altamente influenciada pelo número de células mamárias presentes no úbere, com correlação de 0,50 a 0,73 .

Sabendo que o número total de células na glândula mamária apresenta maior velocidade de crescimento dos dois aos nove meses de idade, antes da puberdade, quando comparado com o aumento da superfície corporal (Sinha \& Tucker, 1969), estudos foram conduzidos objetivando compreender melhor a relação entre diferentes velocidades de desenvolvimento das fêmeas na fase pré-puberdade e seu efeito no desenvolvimento da glândula mamária e como isto interfere na produção de leite, principalmente na primeira lactação. Little \& Kay (1979) e Serjsen et al. (1982), em estudos conduzidos com novilhas de leite, e Buskirk et al. (1996), com novilhas de corte, comentam que a menor produção de leite das novilhas com maiores ganhos de peso até a puberdade é causada pela menor quantidade de parênquima, tecido secretor presente na glândula mamária, e pelos efeitos negativos que a deposição de gordura e obesidade causam no desenvolvimento da glândula mamária. Sobre este último fator, Rovira (1974) acrescenta ainda que o acúmulo de gordura no úbere compete por espaço físico com o tecido secretor, que é o parênquima.

Trabalhos que avaliaram diferentes taxas de ganho de peso no período anterior à puberdade das fêmeas apontam para resultados semelhantes aos do presente estudo. Gardner et al. (1977) relataram reduções na ordem de 10 a $25 \%$ na primeira lactação quando o ganho de peso antes da puberdade ultrapassou $0,8 \mathrm{~kg} /$ dia. Comparando ganhos de 0,4; 0,6 e 0,8 kg/dia de novilhas de diferentes composições raciais até os 184 dias de idade, Ferrell (1982) verificaram maior produção de leite para o ganho intermediário. Johnsson \& Obst (1984) constataram que o nível nutricional antes e após os oito meses de idade pode afetar a produção de leite de novilhas de corte até a terceira lactação, concluindo que novilhas que ganharam $0,55 \mathrm{~kg} / \mathrm{dia}$ antes dos oito meses de idade tiveram maiores produções de leite que aquelas que ganharam 0,67 kg/dia. No trabalho de Buskirk et al. (1996), que compararam dietas com níveis moderado e alto de energia, foram registrados ganhos de peso de 1,03 e 1,34 kg/dia até os 120 dias de idade, respectivamente, sendo que a média de produção de leite (49 aos 161 dias) foi $20 \%$ menor (7,9 vs 6,3 L/dia) para as fêmeas que ganharam mais peso. Avaliando a primeira lactação de novilhas Holandês que ganharam 0,6; 0,8 e 1,0 kg/dia antes da puberdade, Van Amburgh et al. (1998) observaram redução de $5 \%$ na produção de leite quando o ganho passou de 0,6 para $1,0 \mathrm{~kg} /$ dia.

Não houve diferença significativa para produção diária e total $(\mathrm{P}=0,3874)$ de leite entre os tipos de pastagem a que os animais foram submetidos. Contudo, numericamente, vacas mantidas em pastagem cultivada produziram $12 \%$ mais leite $(4,51 \mathrm{~L} / \mathrm{dia})$ que as mantidas em pastagem nativa $(4,03 \mathrm{~L} / \mathrm{dia})$. No trabalho de Restle et al. (2003), que incluiu os dados de 60 vacas, entre elas às 28 do presente estudo, a diferença na produção de leite entre os dois tipos de pastagem foi significativa. Além do menor número de vacas, o presente estudo incluiu a produção de leite das vacas no desmame dos bezerros, o que não foi feito naquele trabalho. Segundo Ribeiro et al. (1991) e Restle et al. (2003), entre os fatores ambientais que interferem na produção de leite, o nível nutricional é o mais importante. Diversos estudos apontam para maior incremento na produção de leite de vacas submetidas a melhores condições nutricionais, principalmente durante o período pós-parto, discordando dos resultados verificados no presente estudo. Estudando a produção de leite de vacas Hereford por três anos consecutivos, Neville Jr. et al. (1974) verificou que as diferenças na produção de leite entre os anos foram resultantes das diferenças na qualidade nutricional da dieta. Restle et al. (2005) relataram que vacas mantidas 
em pastagem nativa produziram $39 \%$ menos leite/dia que as vacas mantidas em pastagem nativa mais pastagem cultivada (3,93 vs 5,46 L/dia, respectivamente).

Para produção de leite, não houve interação significativa entre taxa de ganho de peso na fase inicial do desenvolvimento da vaca e o tipo de pastagem após o parto. A diferença na produção de leite em favor das vacas mantidas em pastagem cultivada em relação às mantidas em pastagem nativa foi de $10 \%$ para as de baixa taxa de ganho de peso e de $14 \%$ para as de moderada taxa de ganho de peso.

Os valores médios referentes ao percentual e à produção total dos componentes do leite, de acordo com os diferentes efeitos avaliados, encontram-se na Tabela 2.

Não houve efeito significativo da taxa de ganho de peso da vaca até os sete meses de idade e do tipo de pastagem após o seu parto sobre a percentagem dos componentes do leite avaliados.

Estudando a produção de leite das novilhas mestiças Angus - Simental com ganhos de 1,03 e 1,34 kg/ dia até os 112 dias de idade, Buskirk et al. (1996) não verificaram diferença na composição do leite até os 161 dias de lactação. Os valores de gordura e extrato seco desengordurado foram, respectivamente, de 2,8 e $8,6 \%$ para o menor ganho e de 2,5 e $8,5 \%$ para o maior ganho. Avaliando diferentes sistemas de alimentação na produção de leite de vacas Charolês e Angus, Ribeiro (1989) verificou leite com percentuais similares de gordura $(3,45 \%)$, lactose $(4,89 \%)$, extrato seco total $(11,7 \%)$ e extrato seco desengordurado $(8,22 \%)$. Em estudo que avaliou a produção e composição do leite de vacas de corte por 90 dias, Senna (1996) reportou menor percentual de gordura e extrato seco total no leite de vacas mantidas exclusivamente em pastagem nativa $(3,0$ e $11,7 \%)$ em relação às mantidas em pastagem cultivada no pré $(3,5$ e 12,2\%) e pós-parto (3,4 e 12,2\%), sendo que para os percentuais de lactose e extrato seco desengordurado não foram verificadas diferenças.

Contudo, para a produção total dos componentes, verifica-se que vacas com menores taxas de ganhos de peso produziram maior quantidade de gordura $(47,4$ vs $35,9 \mathrm{~kg})(\mathrm{P}=0,1055)$, lactose $(52,6$ vs 39,7 $\mathrm{kg}$ ), extrato seco total (140,4 vs $107,2 \mathrm{~kg})$ e extrato seco desengordurado $(94,8 \mathrm{vs} 71,2 \mathrm{~kg})$. As diferenças foram expressivas e na ordem de 32, 32, 31 e 33\%, respectivamente. Os resultados de produção total dos componentes do leite foram diretamente relacionados aos valores da produção de leite (Tabela 1). Entre os diferentes tipos de pastagem, não houve diferença $(\mathrm{P}>0,05)$ na produção total dos componentes avaliados.

$\mathrm{O}$ peso aos sete meses de idade das bezerras com moderada taxa de ganho de peso foi de 130,3 kg, enquanto para as bezerras com baixa taxa de ganho de peso, de 102,4 kg ( $\mathrm{P}<0,05)$; no entanto, quando vacas, o peso ao parto foi similar $(\mathrm{P}>0,05)$, de 366,7 e $371,8 \mathrm{~kg}$, respectivamente. Pode-se afirmar que taxas de ganho inferiores a $350 \mathrm{~g} /$ dia até os sete meses de idade não foram prejudiciais ao crescimento futuro das bezerras, em razão da compensação no peso que estas apresentaram na fase pós-desmama. Restle et al. (1999c) verificaram que bezerros com menores taxas de ganho de peso durante o aleitamento e menor peso ao desmame, compensaram a diferença no período subsequiente ao desmame, resultando em pesos similares aos dois anos de idade.

Para o peso da vaca por ocasião do desmame de seus bezerros $(397,8$ e $411,7 \mathrm{~kg}$ ) e ganho de peso médio diário do parto ao desmame (143 e 184 g), também houve similaridade $(\mathrm{P}>0,05)$, respectivamente, para as taxas de ganho de peso moderada e baixa na fase inicial da vida das vacas.

Em relação aos tipos de pastagem, vacas mantidas em pastagem cultivada apresentaram maior $(\mathrm{P}<0,05)$ ganho de peso médio diário que as mantidas em pastagem nativa (342 vs $-15 \mathrm{~g} / \mathrm{dia}$ ), demonstrando as diferentes maneiras como os nutrientes podem ser aproveitados pelos animais de acordo com sua disponibilidade. Nas vacas mantidas em pastagem cultivada, o excesso de nutrientes não foi utilizado para incrementar a produção de leite (Tabela 1) e sim para ganho de peso e provavelmente para o acúmulo de gordura corporal. Nos animais que apresentaram baixas taxas de ganho até os sete meses de idade, houve prioridade para produção de leite (Tabela 1), sem causar perdas de peso. Holloway et al. (1985) acrescentam que a prioridade do uso de nutrientes também pode variar entre os grupos genéticos, de acordo com suas diferenças nos requerimentos de energia para as várias funções corporais.

$\mathrm{Na}$ Tabela 3, estão apresentadas as médias referentes ao desempenho dos bezerros até o desmame, de acordo com a taxa de ganho de peso da vaca até os sete meses de idade e com o tipo de pastagem.

O peso do bezerro ao nascer não foi influenciado pela taxa de ganho de peso da mãe na fase de aleitamento. Contudo, o peso ao desmame foi $14 \%$ 
Tabela 2 - Médias para percentagens e produção total dos componentes do leite de acordo com a taxa de ganho de peso da vaca em seu período pré-desmame e com o tipo de pastagem durante a lactação

Table 2 - Means for percentages and total production of milk components according to cow rate of weight gain in their preweaning period and with pasture during their lactation

\begin{tabular}{|c|c|c|c|c|}
\hline \multirow[t]{2}{*}{$\begin{array}{l}\text { Pastagem } \\
\text { Pasture }\end{array}$} & \multicolumn{4}{|c|}{$\begin{array}{c}\text { Taxa de ganho de peso quando bezerra } \\
\text { Rate of weight gain as heifer calf }\end{array}$} \\
\hline & \multicolumn{2}{|c|}{$\begin{array}{l}\text { Moderada } \\
\text { Moderate }\end{array}$} & \multicolumn{2}{|c|}{$\begin{array}{c}\text { Baixa } \\
\text { Low }\end{array}$} \\
\hline & \multicolumn{4}{|c|}{$\begin{array}{c}\text { Gordura } \\
\text { Fat }\end{array}$} \\
\hline & $\%$ & $\mathrm{~kg}$ & $\%$ & $\mathrm{~kg}$ \\
\hline Nativa(Native) & 4,11 & 32,6 & 4,57 & 46,0 \\
\hline Cultivada (Cultivated) & 4,49 & 39,2 & 4,30 & 48,9 \\
\hline \multirow[t]{3}{*}{ Média (Mean) } & 4,30 & $35,9 \mathrm{~B}$ & 4,43 & $47,4 \mathrm{~A}$ \\
\hline & \multicolumn{4}{|c|}{$\begin{array}{l}\text { Lactose } \\
\text { Lactose }\end{array}$} \\
\hline & $\%$ & $\mathrm{~kg}$ & $\%$ & $\mathrm{~kg}$ \\
\hline Nativa(Native) & 4,85 & 36,1 & 5,08 & 50,5 \\
\hline Cultivada (Cultivated) & 5,07 & 43,2 & 5,01 & 54,8 \\
\hline \multirow[t]{3}{*}{ Média (Mean) } & 4,96 & $39,7 \mathrm{~B}$ & 5,04 & $52,6 \mathrm{~A}$ \\
\hline & \multicolumn{4}{|c|}{$\begin{array}{l}\text { Extrato seco total } \\
\text { Total dry extract }\end{array}$} \\
\hline & $\%$ & $\mathrm{~kg}$ & $\%$ & $\mathrm{~kg}$ \\
\hline Nativa(Native) & 12,83 & 98,2 & 13,15 & 136,1 \\
\hline Cultivada(Cultivated) & 13,46 & 116,2 & 12,99 & 144,7 \\
\hline \multirow[t]{3}{*}{ Média (Mean) } & 13,15 & $107,2 \mathrm{~B}$ & 13,07 & $140,4 \mathrm{~A}$ \\
\hline & \multicolumn{4}{|c|}{$\begin{array}{c}\text { Extrato seco desengordurado } \\
\text { Nonfat dry extract }\end{array}$} \\
\hline & $\%$ & $\mathrm{~kg}$ & $\%$ & $\mathrm{~kg}$ \\
\hline Nativa(Native) & 8,72 & 65,4 & 8,82 & 91,7 \\
\hline Cultivada (Cultivated) & 8,96 & 76,9 & 8,87 & 97,8 \\
\hline Média (Mean) & 8,84 & $71,2 \mathrm{~B}$ & 8,85 & $94,8 \mathrm{~A}$ \\
\hline
\end{tabular}

superior para os bezerros produzidos pelas vacas com menores taxas de ganho de peso (189,0 vs $166,1 \mathrm{~kg})$. Os resultados de ganho de peso médio diário e peso à desmama em parte são explicados pelas variações na produção de leite das vacas (Tabela 1), sendo confirmado pelos coeficientes de correlação positivos entre peso ao desmame e ganho de peso do nascimento ao desmame com produção de leite das vacas (Tabela 5). Diversos estudos relatam correlação positiva entre produção de leite e ganho de peso dos bezerros (Neville Jr., 1962; Rutledge et al., 1971; Totusek et al., 1973; Robison et al., 1978; Alencar et al., 1985; Ribeiro \& Restle, 1991).

Consta, na Tabela 3, a influência que o tipo de pastagem exerceu sobre o ganho de peso do bezerro durante o período de aleitamento e sobre o peso ao desmame. Bezerros mantidos com as mães em pastagem cultivada apresentaram peso ao desmame $55 \%$ superior aos mantidos em pastagem nativa. Os estudos de Costa et al. (1981) e Moojen et al. (1994) confirmam os resultados verificados no presente estudo, demonstrando vantagem no desempenho dos animais manejados em pastagem cultivada.

A diferença para os bezerros mantidos em pastagem cultivada em relação aos mantidos em pastagem nativa foi de $82 \%$ no ganho de peso médio diário do nascimento ao desmame e de $66 \%$ no peso ao desmame quando as mães apresentaram baixa taxa de ganho de peso, e de 63 e $44 \%$, respectivamente, quando as mães apresentaram moderada taxa de ganho de peso. Embora para o desempenho do bezerro a interação entre tipo de pastagem e taxa de ganho de peso na fase inicial da vida da vaca não tenha sido significativa, verifica-se que o benefício da pastagem cultivada foi mais evidente nos bezerros cujas mães tiveram menor taxa de ganho de peso. É provável que o maior 
Tabela 3 - Médias para características de desempenho dos bezerros de acordo com a taxa de ganho de peso da vaca em seu período pré-desmame e com o tipo de pastagem durante a lactação

Table 3 - Means for characteristics of performance of calves according to cow rate of weight gain in their preweaning period and with pasture during their lactation

\begin{tabular}{|c|c|c|}
\hline \multirow[t]{2}{*}{$\begin{array}{l}\text { Pastagem } \\
\text { Pasture }\end{array}$} & \multicolumn{2}{|c|}{$\begin{array}{c}\text { Taxa de ganho de peso quando bezerra } \\
\text { Rate of weight gain as heifer calf }\end{array}$} \\
\hline & $\begin{array}{l}\text { Moderada } \\
\text { Moderate }\end{array}$ & $\begin{array}{l}\text { Baixa } \\
\text { Low }\end{array}$ \\
\hline & \multicolumn{2}{|c|}{$\begin{array}{c}\text { Peso ao nascer do bezerro, } \mathrm{kg}^{*} \\
\text { Calf birth weight, } \mathrm{kg} *\end{array}$} \\
\hline & $32,3 \mathrm{~A}$ & $33,4 \mathrm{~B}$ \\
\hline & \multicolumn{2}{|c|}{$\begin{array}{l}\text { Peso ao desmame do bezerro, } \mathrm{kg} \\
\text { Calf weaning weight, } \mathrm{kg}\end{array}$} \\
\hline Nativa(Native) & 136,1 & 142,0 \\
\hline Cultivada(Cultivated) & 196,1 & 235,9 \\
\hline \multirow[t]{2}{*}{ Média (Mean) } & $166,1 \mathrm{~B}$ & $189,0 \mathrm{~A}$ \\
\hline & \multicolumn{2}{|c|}{$\begin{array}{c}\text { Ganho médio diário do nasc. ao desmame, } \mathrm{g} \\
\text { Average daily gain from birth to weaning, } g\end{array}$} \\
\hline Nativa(Native) & 469 & 508 \\
\hline Cultivada (Cultivated) & 764 & 926 \\
\hline Média (Mean) & 617B & $717 \mathrm{~A}$ \\
\hline
\end{tabular}

desenvolvimento dos bezerros, causado pela maior produção de leite destas vacas, tenha resultado também em maiores exigências para mantença e ganho de peso (NRC, 1996), estimulando o consumo de pasto, acarretando maior aporte de nutrientes e favorecendo o seu desenvolvimento.

Em condições de pastagem cultivada, o ganho de peso médio diário e o peso ao desmame dos bezerros filhos de vacas com baixo ganho de peso inicial foram superior aos dos filhos de vacas com ganho de peso moderado, em 21 e $20 \%$, respectivamente.

Em condições de pastagem nativa, a diferença foi de 8 e 4\%, respectivamente. Portanto, o melhor desempenho dos bezerros filhos de vacas com menor taxa de ganho de peso inicial, mantidos em condições de pastagem cultivada, foi efeito da maior produção de leite das mães associado à melhor qualidade da pastagem (Tabela 3). A menor diferença em condições de pastagem nativa foi decorrente da baixa qualidade da pastagem, da digestibilidade e do teor de proteína baixos, o que deve ter reduzido o consumo de matéria seca e o aporte de nutrientes e, conseqüentemente, limitado o desenvolvimento dos bezerros.

Encontram-se, na Tabela 4, os coeficientes de correlação de Pearson entre as características estu- dadas de acordo com as taxas de ganhos de peso das vacas até os sete meses de idade e, na Tabela 5, as correlações gerais entre as características.

Analisando a correlação entre a produção de leite, o peso dos bezerros à desmama e o ganho de peso médio diário dos bezerros até o desmame, verifica-se maior valor quando as taxas de ganhos de peso na fase inicial da vida das vacas foram maiores $(r=0,46$ e 0,18 , respectivamente) (Tabela 4). Como a produção de leite foi menor nas vacas com moderada taxa de ganho (Tabela 1), pequenas variações no aumento da produção resultaram em maior resposta no desempenho dos bezerros, enquanto, para os bezerros filhos de vacas com baixas taxas de ganho, houve menor sensibilidade às oscilações na produção de leite das vacas, uma vez que a disponibilidade de leite para o bezerro foi maior, preenchendo maior parte de seus requerimentos nutricionais.

Analisando os coeficientes de correlação gerais (Tabela 5), nota-se que o peso e o ganho de peso dos bezerros se correlacionaram positivamente com a produção de leite da vaca ( $\mathrm{r}=0,30$ e 0,27 , respectivamente). Vários trabalhos [Neville Jr., 1962 (0,60); Melton et al., 1967 (0,40); Rutledge et al., 1971 (0,60); Chenette \& Frahm, 1981 (0,20); Ribeiro \& Restle, $1991(0,75)$; 


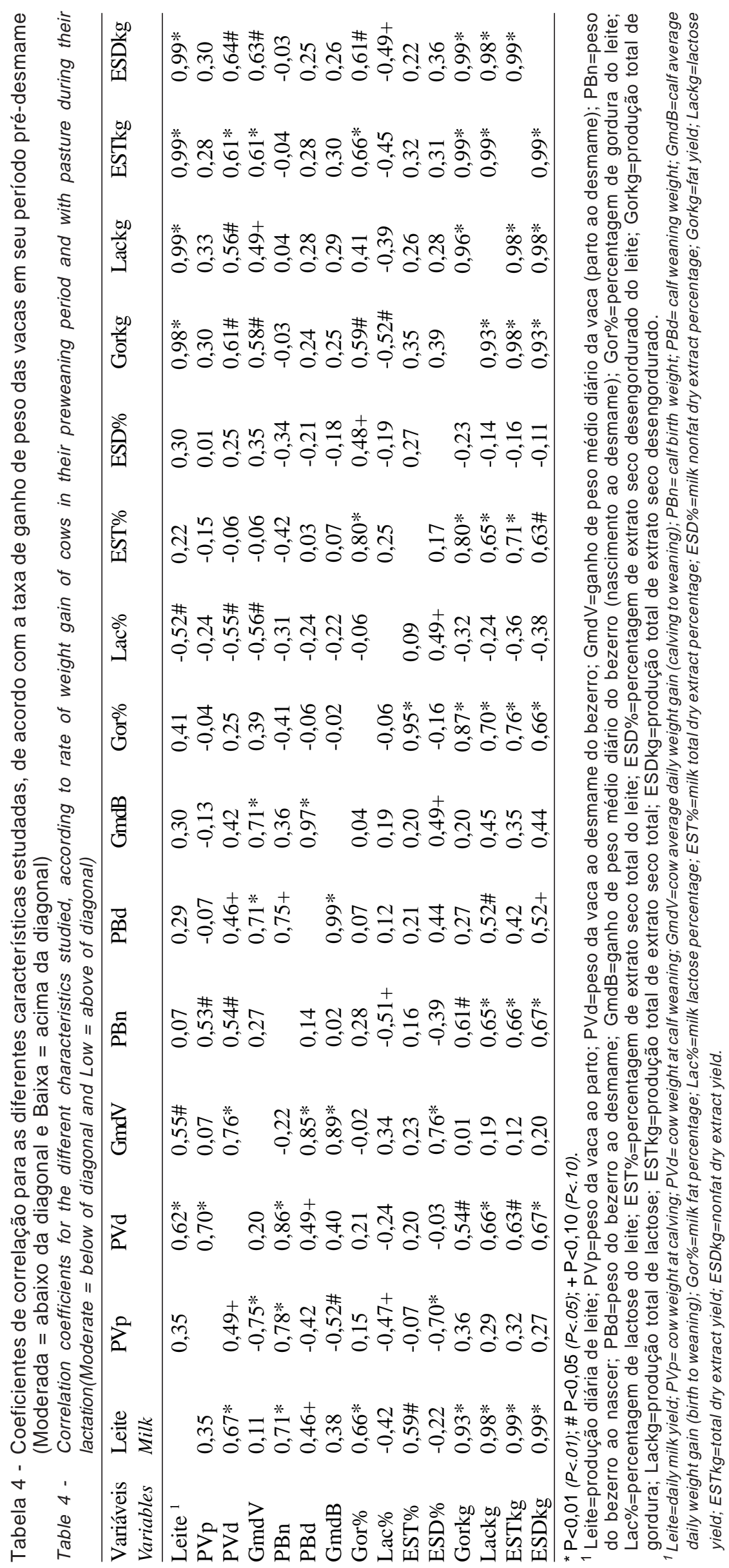


Albuquerque et al., $1993(0,71)]$ reportam resultados similares aos deste estudo, confirmando a importância da produção de leite no desenvolvimento inicial do bezerro até o desmame.

As correlações entre os percentuais dos componentes do leite e o desempenho dos bezerros foram baixas (algumas próximas de zero) e variaram de $-0,06$ e 0,21 . Contudo, para a produção total dos componentes, as correlações com o peso ao desmame e os ganhos de peso médio diários dos bezerros foram maiores, apresentando valores semelhantes aos resultados verificados para a produção de leite, porque houve elevada relação entre produção de leite e produção total dos componentes do leite.

Ribeiro \& Restle et al. (1991) verificaram que as porcentagens médias dos componentes do leite tiveram correlações muito baixas e não-significativas, tanto com os pesos como com os ganhos de peso médio diários dos bezerros. Porém, as produções destes componentes tiveram correlações altas e significativas com o ganho de peso médio diário do nascimento ao desmame. Resultados semelhantes foram relatados por Melton et al. (1967), Rutledge et al. (1971) e Totusek et al. (1973).

Entre os pesos das vacas e a produção de leite (Tabela 5), a relação foi positiva, ou seja, quanto maior o peso da vaca ao parto e principalmente ao desmame, maior a produção de leite. Os coeficientes de correlação foram de 0,34 e 0,60, respectivamente. Além disso, os pesos das vacas correlacionaram-se positivamente com os pesos dos bezerros: quanto maior o peso da vaca ao parto, maior o peso do bezerro ao nascer $(r=0,68)$ e, quanto maior o peso da vaca ao desmame, maior o peso do bezerro ao desmame $(r=0,47)$. Segundo Ribeiro et al. (1991) e Restle et al. (2003), este resultado está associado à maior produção de leite das vacas de maior tamanho corporal. Correlação positiva entre peso ao nascer do bezerro e peso ao parto de vacas Canchim foi relatada por Alencar et al. (1996).

Associação positiva também foi verificada entre a produção de leite e o peso do bezerro ao nascer $(\mathrm{r}=0,28)$ por Alencar et al. (1985), que verificaram que, quanto maior o peso do bezerro ao nascer, maior a produção de leite da vaca, apresentando coeficiente de regressão linear de 0,082. Trabalhando com vacas da raça holandesa, Chew et al. (1981), citados por Alencar et al. (1985), verificaram esses mesmos efeitos, porém mais acentuados no início da lactação. Como em gado de leite o bezerro é separado da vaca nas primeiras horas de vida, os autores concluíram que o peso do bezerro ao nascimento pode estar relacionado à produção de hormônios ligados à lactogênese. Em gado de corte, além do aspecto hormonal, Alencar et al. (1985) ressaltam que o peso do bezerro pode influenciar a produção de leite por sua capacidade ou necessidade de mamar mais. Rutledge et al. (1971) observaram regressão linear entre produção total de leite das vacas e peso ao nascer dos bezerros, indicando que bezerros mais pesados ao nascimento demandaram mais leite de suas mães ou tiveram maior capacidade para consumir leite. Correlações de 0,43 e 0,29 entre peso ao nascer e produção de leite durante o primeiro e terceiro mês de lactação foram observadas por Drewry et al. (1959).

Analisando a relação entre os percentuais dos componentes do leite e a produção de leite, nota-se que houve associação positiva para gordura $(\mathrm{r}=0,49)$ e extrato seco total $(\mathrm{r}=0,31)$ e negativa para lactose $(\mathrm{r}=-0,35)$ (Tabela 5). Correlação positiva entre a produção diária de leite e a porcentagem de gordura e extrato seco total foram obtidas por Melton et al. (1967) e Chenette \& Frahm (1981). No entanto, trabalhos apontam para relação negativa entre produção de leite e percentagem de gordura (Lamond et al., 1969; Rutledge et al., 1971; Schmidt \& Van Vleck, 1976; Richardson et al., 1977; Maynard et al., 1984), em virtude do aumento da concentração deste componente com a diminuição do volume de leite produzido.

Correlação positiva também foi verificada entre percentagem de gordura e percentagem de extrato seco total $(r=0,89)$. A variação no teor de extrato seco total é resultado da oscilação do somatório dos demais componentes do leite, principalmente a gordura, que apresenta maior proporção. Outros estudos relatam relação positiva entre estes dois componentes, como os de Wilson et al. (1969) (0,60), Chenette \& Frahm (1981) $(0,36)$ e Cruz et al. (1997) $(0,94)$. 


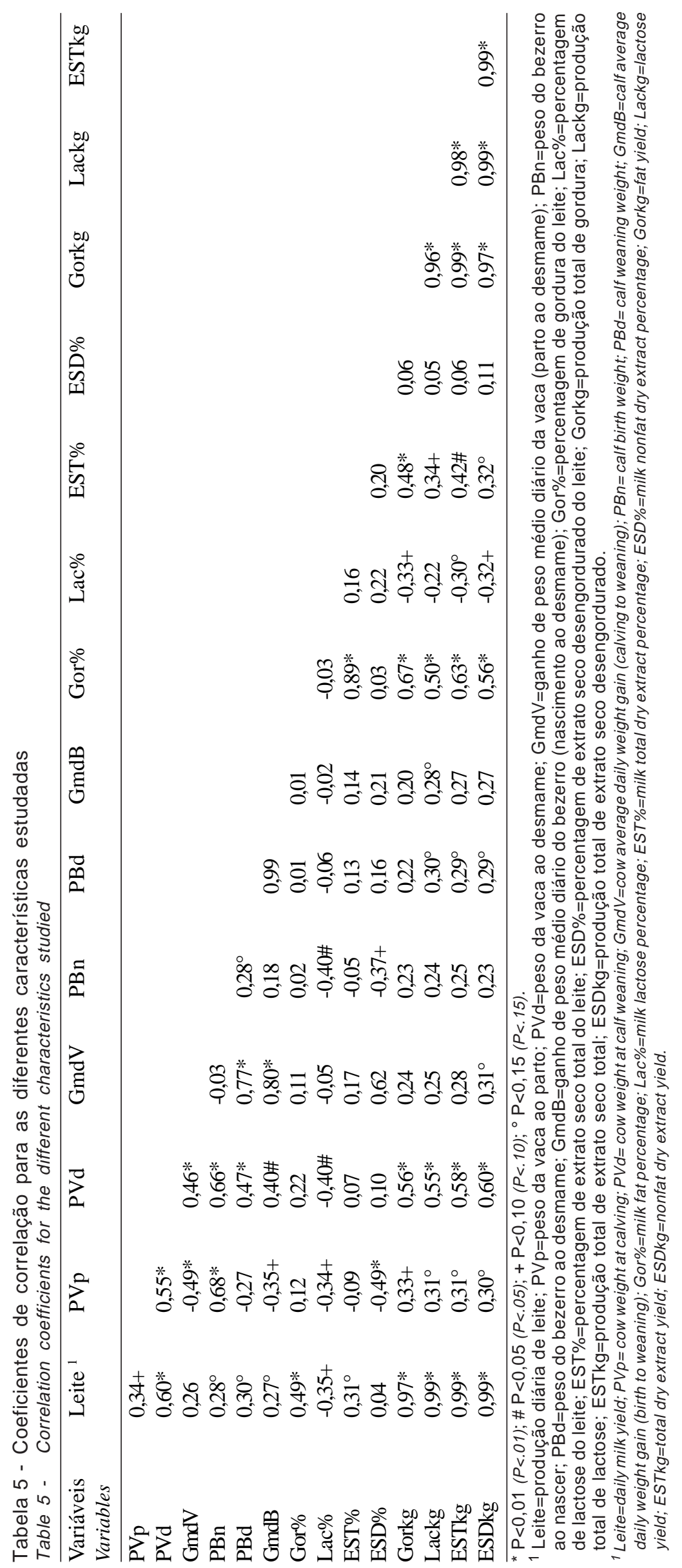




\section{Conclusões}

Taxas de ganho de peso inferiores a $350 \mathrm{~g} /$ dia até os sete meses de idade não foram prejudiciais ao crescimento futuro das bezerras, em razão da compensação no peso que apresentaram na fase pós-desmama.

Bezerras com taxas de ganho de peso inferiores a $350 \mathrm{~g} /$ dia até os sete meses de idade, quando vacas, produziram mais leite, maior quantidade dos componentes do leite e bezerros mais pesados ao desmame que aquelas que apresentaram taxas de ganhos de peso acima de $350 \mathrm{~g} / \mathrm{dia}$.

\section{Literatura Citada}

ALBUQUERQUE, L.G.; ELER, J.P.; COSTA, M.J.R.P. Produção de leite e desempenho do bezerro na fase de aleitamento em três raças bovinas de corte. Revista da Sociedade Brasileira de Zootecnia, v.22, n.5, p.745-754, 1993.

ALENCAR, M.M.; FILHO, A.A.J.; PARANHOS, N.E. Produção de leite das vacas e desenvolvimento dos bezerros em um rebanho Canchim. In: REUNIÃO ANUAL DA SOCIEDADE BRASILEIRA DE ZOOTECNIA, 22., Camboriú. Anais... Viçosa, MG: Sociedade Brasileira de Zootecnia, 1985. p. 223.

ALENCAR, M.M.; TULLIO, R.R.; CRUZ, G.M. et al. Produção de leite da vaca e desenvolvimento do bezerro em gado de corte. Revista da Sociedade Brasileira de Zootecnia, v.25, n.1, p.92-101, 1996.

ASSOCIATION OF OFFICIAL ANALYTICAL CHEMISTS AOAC. Official methods of analysis. 14.ed. Washington, D.C.: 1984. 1141p.

BUSKIRK, D.D.; FAULKNER, D.B.; HURLEY, W.L. et al. Growth, reproductive performance, mammary development, and milk production of beef heifers as influenced by prepubertal dietary energy and administration of bovine somatotropin. Journal of Animal Science, v.74, p.2649-2662, 1996.

CHENETTE, C.G.; FRAHM, R.R. Yield and composition of milk from various two-breed cross cows. Journal of Animal Science, v.52, n.3, p.483-492, 1981.

COSTA, A.M.; RESTLE, J.; MÜLLER, L. Influência da pastagem cultivada no desempenho reprodutivo de vacas com cria ao pé. Revista do Centro de Ciências Rurais, v.11, n.4, p.187-200, 1981.

CRUZ, G.M.; ALENCAR, M.M.; TULliO, R.R. Produção e composição do leite de vacas das raças Canchim e Nelore. Revista Brasileira de Zootecnia, v.26, n.5, p.887-893, 1997.

DREWRY, K.J.; BROWN, C.J.; HONEA, R.S. Relationships among factors associated with mothering ability in beef cattle. Journal of Animal Science, v.18, p.938-946, 1959.

FERRELL, C.L. Effects of postweaning rate of gain on onset of puberty and productive performance of heifers of different breeds. Journal of Animal Science, v.55, p.12721283, 1982.

GARDNER, R.W.; SCHUM, J.D.; VARGUS, L.G. Accelerated growth and early breeding of Holstein heifers. Journal of Dairy Science, v.60, p.1941-1948, 1977.
HACKETT, A.J.; TUCKER, H.A. Prediction of mammary nucleic acid content and lactational performance from measurements during immaturity. Journal of Dairy Science, v.51, p.957 (Abstract), 1968.

HOLLOWAY, J.W.; BUTTS, W.T.; McCURLEY, J.R. et al. Breed $\mathrm{x}$ nutritional environment interactions for beef female weight and fatness, milk production and calf growth. Journal of Animal Science, v.61, n.6, p.1354-1363, 1985.

JOHNSSON, I.D.; OBST, J.M. The effect of level of nutrition before and after 8 months of age on subsequent milk and calf production of beef heifers over three lactations. Animal Production, v.38, n.3, p.57-68, 1984.

LAMOND, D.R.; HOLMES, J.H.G.; HAYDOCK, K.P. Estimation of yield and composition of milk produced by grazing beef cows. Journal of Animal Science, v.29, p.606-611, 1969.

LITTLE, W.; KAY, R.M. The effects of rapid rearing and early calving on the subsequent performance of dairy heifers. Animal Production, v.29, p.131-142, 1979.

MAYNARD, L.A.; LOOSLI, J.K.; HINTZ, H.F. et al. Nutrição animal. 3.ed. Rio de Janeiro: Freitas Bastos, 1984. 736p.

MELTON, A.A.; RIGGS, J.K.; NELSON, L.A. et al. Milk production, composition and calf gains of Angus, Charolais and Hereford cows. Journal of Animal Science, v.26, p.804-809, 1967.

MOOJEN, J.L.; RESTLE, J.; MOOJEN, E.L. et al. Efeito da época da desmama e da pastagem no desempenho de vacas e terneiros de corte. 1. Desempenho de terneiros. Ciência Rural, v.24, n.2, p.399-403, 1994.

MORENO, J.A. Clima do Rio Grande do Sul. Porto Alegre: Secretaria da Agricultura, 1961. 42p.

NATIONAL RESEARCH COUNCIL - NRC. Nutrient requirements of beef cattle. 6.ed. Washington, D.C.: National Academy Press, 1996. 242p.

NEVILLE JR., W.E. The influence of dam's milk production and other factors on 120- and 240-day weight of Hereford calves. Journal of Animal Science, v.21, p.315, 1962.

NEVILLE JR., W.E.; WARREN, E.P.; GRIFFEY, W.A. Estimates of age effects on milk production in Hereford cows. Journal of Animal Science, v.38, v.1, p.1-5, 1974.

QUADROS, S.A.F.Q.; LOBATO, J.F.P. Efeitos da lotação animal na produção de leite de vacas de corte primíparas e no desenvolvimento de seus bezerros. Revista Brasileira de Zootecnia, v.26, n.1, p.27-33, 1997.

RESTLE, J.; BRONDANI, I.L.; BERNARDES, R.A.C. O novilho superprecoce. In: RESTLE, J. (Ed.) Confinamento, pastagens e suplementação para produção de bovinos de corte. Santa Maria: Universidade Federal de Santa Maria, 1999b. p.191-214.

RESTLE, J.; PACHECO, P.S.; MOLETTA, J.L. et al. Grupo genético e nível nutricional pós-parto na produção e composição do leite de vacas de corte. Revista Brasileira de Zootecnia, v.32, n.3, p.585-597, 2003.

RESTLE, J.; POLLI, V.A.; ALVES FILHO, D.C. et al. Desenvolvimento de bovinos de corte de diferentes grupos genéticos desmamados aos 3 ou 7 meses de idade. Revista Brasileira de Zootecnia, v.28, n.5, p.1023-1030, 1999c.

RESTLE, J.; POLLI, V.A.; SENNA, D.B. Efeito de grupo genético e heterose sobre a idade e peso à puberdade e sobre o desempenho reprodutivo de novilhas de corte. Pesquisa Agropecuária Brasileira, v.34, n.4, p.701-707, 1999a. 
RESTLE, J.; SENNA, D.B.; PACHECO, P.S. et al. Grupo genético e heterose na produção de leite de vacas de corte. Revista Brasileira de Zootecnia, 2005 (prelo).

RIBEIRO, E.L.A. Influência de diferentes seqüências de pastagens na produção de leite e no desempenho de vacas de dois grupos genéticos. Santa Maria: Universidade Federal de Santa Maria, 1989. 149p. Dissertação (Mestrado em Zootecnia) - Universidade Federal de Santa Maria, 1989.

RIBEIRO, E.L.A.; RESTLE, J. Desempenho de terneiros Charolês e Aberdeen Angus puros e seus mestiços com Nelore. Pesquisa Agropecuária Brasileira, v.26, n.8, p.1145-1151, 1991.

RIBEIRO, E.L.A.; RESTLE, J.; PIRES, C.C. Produção e composição do leite de vacas Charolês e Aberdeen Angus amamentando bezerros puros ou mestiços. Pesquisa Agropecuária Brasileira, v.26, n. 8, p.1267-1273, 1991.

RICHARDSON, F.D.; OLIVER, J.; CLARKE, G.P.Y. Analysis of some factors with affect the productivity of beef cows and of their calves in a marginal rainfall area of Rhodesia. 2. The yield and composition of milk of suckling cows. Animal Production, v.25, p.359-372, 1977.

RIO GRANDE DO SUL. Secretaria da Agricultura. Departamento de Pesquisa. Instituto de Pesquisas Agronômicas. Observações Meteorológicas do Estado do Rio Grande do Sul. Boletim Técnico, n.3, 1979, 270p.

ROBISON, O.W.; YUSUFF, M.K.M.; DILLARD, E.U. Milk production in Hereford cows I. Means and correlations. Journal of Animal Science, v.47, n.1, p.131-135, 1978.

ROVIRA, J. Reproduccion y manejo de los rodeos de cria. Montevideo: Hemisferio Sur. 1974. 293p.

RUTLEDGE, J.J.; ROBISON, O.W.; AHLSCHWEDE, W.T. et al. Milk yield and its influence on 205-day weight of beef calves. Journal of Animal Science, v.33, p.563-567, 1971.

STATISTICAL ANALYSIS SYSTEMS - SAS. User's guide. Version 6, Cary: v.2, 1997. 1052p.

SCHMIDT, G.H.; Van VLECK, L.D. Bases científicas de la producción lechera. Zaragoza: Acríbia, 1976. 583p.

SERJSEN, K.; HUBER, T.J.; TUCKER, H.A. Influence of nutrition on mammary development in pre- and postpubertal heifers. Journal of Dairy Science, v.65, p.793-800, 1982.
SENNA, D.B. Desempenho reprodutivo e produção de leite de vacas de quatro grupos genéticos, desterneiradas precocemente, submetidas a diferentes períodos de pastagem cultivada. Santa Maria: Universidade Federal de Santa Maria, 1996. 85p. Dissertação (Mestrado em Zootecnia) Universidade Federal de Santa Maria, 1996.

SINHA, Y.N.; TUCKER, H.A. Mammary development and pituitary prolactin level of heifers from birth through puberty and during the estrous cycle. Journal of Dairy Science, v.52, p.507-512, 1969.

TILLEY, J.M.A.; TERRY, R.A. A two-stage technique for the in vitro digestion of forage crop. Journal of British Grassland Society, v.18, n.2, p,104-111, 1963.

TOTUSEK, R.; ARNETT, D.W.; HOLLAND, G.L. et al. Relation of estimation method, sampling interval and milk composition to milk yield of beef cows and calf gain. Journal of Animal Science, v.37, n.1, p.153-158, 1973.

VAN AMBURGH, M.E.; GALTON, D.M.; BAUMAN, D.E. et al. Effects of three prepubertal body growth rates on performance of Holstein heifers during first lactation. Journal of Dairy Science, v.81, n.2, p.527-538, 1998.

WILM, H.G.; COSTELlO, D.F.; KLIPPLE, G.E. Estimating forage yield by the double-sampling methods. Journal of American Society of Agronomy, v.36, p.194-203, 1944.

WILSON, L.L.; GILLOOLY, J.E.; RUGH, M.C. et al. Effects of energy intake, cow body size and calf sex on composition and yield of milk by Angus-Holstein cows and preweaning growth rate of progeny. Journal of Animal Science, v.28, p.789, 1969. 